\title{
BMJ Open Conjunctival colonisation and antibiotic resistance of coagulase-negative Staphylococcus after cataract surgery: a 6-month longitudinal study at a medical centre in Taiwan
}

\author{
Eugene Yu-Chuan Kang, ${ }^{1,2}$ Chiun-Ho Hou, ${ }^{1,2}$ Yhu-Chering Huang, ${ }^{2,3}$ \\ Ching-Hsi Hsiao ${ }^{1,2}$
}

To cite: Kang EY-C, Hou C-H, Huang Y-C, et al. Conjunctival colonisation and antibiotic resistance of coagulasenegative Staphylococcus after cataract surgery: a 6-month longitudinal study at a medical centre in Taiwan. BMJ Open 2019;9:e027036. doi:10.1136/ bmjopen-2018-027036

- Prepublication history and additional material for this paper are available online. To view these files, please visit the journal online (http://dx.doi. org/10.1136/bmjopen-2018027036 )

Received 7 0ctober 2018

Revised 7 May 2019

Accepted 20 May 2019

Check for updates

(c) Author(s) (or their employer(s)) 2019. Re-use permitted under CC BY-NC. No commercial re-use. See rights and permissions. Published by BMJ.

${ }^{1}$ Department of Ophthalmology, Chang Gung Memorial Hospital, Linkou Medical Center, Taoyuan, Taiwan

${ }^{2}$ College of Medicine, Chang Gung University, Taoyuan,

Taiwan

${ }^{3}$ Division of Pediatric Infectious Diseases, Department of Pediatrics, Chang Gung Memorial Hospital, Linkou Medical Center, Taoyuan, Taiwan

Correspondence to

Dr Ching-Hsi Hsiao;

hsiao.chinghsi@gmail.com

\section{ABSTRACT}

Objective To explored 6-month longitudinal changes in conjunctival colonisation and antibiotic resistance profiles of coagulase-negative Staphylococcus (CNS) after cataract surgery with 1 month tobramycin treatment.

Design Prospective cohort study between 1 August 2012, and 31 July 2013.

Setting A single medical centre in Taiwan.

Participants A total of 128 Taiwanese patients with $46.9 \%$ of male participants.

Interventions Samples from the conjunctival sacs of both operation (OP) and non-OP eyes were obtained separately before cataract surgery and at 1, 3 and 6 months after surgery. Tobramycin $(0.3 \%)$ treatment was applied four times daily for 1 month postoperatively.

Main outcome measure Identification of CNS isolates and their antibiotic susceptibility by using disk diffusion or E-test.

Results CNS was detected in $24.2 \%$ of patients at baseline. During postoperative follow-up, the CNS colonisation rate did not decrease in either eye but showed an increasing trend in the OP eyes at 1 month $(p=0.06)$. The colonisation rate showed no significant difference between the OP and non-OP eyes from baseline to a specific follow-up. We observed a significant increase $(p<0.05)$ in resistance to tobramycin at 1 month and to ciprofloxacin at 3 months in the OP eyes and to trimethoprim/sulfamethoxazole at 1 month and 3 months and to oxacillin at 6 months in the non-OP eyes.

Conclusions During the 6-month postoperative followup, $0.3 \%$ tobramycin administration failed to reduce CNS colonisation but increased resistance to several antibiotics. Postoperative antibiotic treatment may be replaced by other evidence-endorsed prophylactic routines.

\section{INTRODUCTION}

Cataract surgery is one of the most commonly performed ocular surgeries by ophthalmologists. Postoperative endophthalmitis has been reported as one of the vision-threatening complications after cataract surgery for more than 50 years, ${ }^{1}$ and the overall rate

\section{Strengths and limitations of this study}

- Sampling for both the eyes separately to provide an internal control from the non-operation eyes was performed.

- Susceptibility tests for several antibiotics for ophthalmic use were demonstrated.

- For longitudinal evaluation, patients were followed until 6 months after cataract surgery in the study.

- The small number of cases and low bacterial growth rate in the study may have affected statistical significance.

- Part of participants missed the clinical follow-up during the study period.

was approximately $0.13 \% .^{2}$ Although its prevalence rate is low, postoperative endophthalmitis usually leads to a poor visual outcome. ${ }^{3}$ Therefore, several prophylactic routines have been discussed for endophthalmitis prevention after surgery. ${ }^{45}$

Postoperative antibiotic administration is one of the prophylactic routines for preventing postcataract extraction endophthalmitis. A 2014 online survey conducted in the USA showed that $97 \%$ of ophthalmologists used postoperative topical antibiotics as a prophylactic routine. ${ }^{6}$ Frequently used ophthalmic antibiotics include fluoroquinolones, polymyxin $\mathrm{B} /$ trimethoprim, tobramycin and neomycin. ${ }^{7}$ Fluoroquinolones have been the most commonly studied ophthalmic antibiotics. ${ }^{8-10}$ Although the topical use of fluoroquinolones after cataract surgery might reduce the rate of endophthalmitis, ${ }^{7}$ long-term or repeated exposure to the antibiotics increases the resistance of conjunctival flora. ${ }^{8-10}$

The most frequent organism involved in postoperative endophthalmitis is 
coagulase-negative Staphylococcus (CNS), ${ }^{11}$ which is also the most common flora inhabiting the conjunctival sac according to previous investigations and our study. ${ }^{12-14}$ According to Speaker et $a l \mathrm{~s}^{15}$ investigation, $82 \%$ of isolates from the vitreous samples of patients with endophthalmitis were identical to the organisms isolated from their eyelid, conjunctiva, or nose. These findings indicated the strong correlation between conjunctival CNS colonisation and subsequent ocular infection.

In our hospital, the currently performed prophylactic routines are preoperative povidone-iodine sterilisation and postoperative tobramycin administration. Longitudinal changes in CNS colonisation and its antibiotic resistance have not been effectively analysed before and after cataract surgeries when using tobramycin as a postoperative antibiotic. Therefore, we investigated the effects of postoperative tobramycin administration on CNS colonisation and antibiotic resistance in both the eyes of patients undergoing cataract surgery to determine implications for general practice at Chang Gung Memorial Hospital $(\mathrm{CGMH})$ in Taiwan.

\section{PATIENTS AND METHODS \\ Patient enrolment}

Between 1 August 2012 and 31 July 2013, participants aged >18years scheduled for cataract surgery with phacoemulsification and intraocular lens implantation by a single surgeon were recruited. Patients who were pregnant, had active eye infection or used topical or systemic antibiotics within 3 months before the operation were excluded. After the operation, TobraDex $(0.3 \%$ tobramycin $+0.1 \%$ dexamethasone) ophthalmic suspension was prescribed to every patient's operation eye and applied four times a day for 1 month. This study adhered to the Declaration of Helsinki.

\section{Data collection}

The study purpose and sampling method were explained to every patient before obtaining informed consent. Every patient received a questionnaire that consisted of history of hospitalisation, operation, dialysis, medication use (antibiotics or immunosuppressants), sociodemographic information (alcohol abuse, homelessness and close contact with children younger than 5 years) and medical comorbidities (skin disease, HIV infection, liver abscess, diabetes mellitus, cardiac disease, malignancy, chronic kidney disease and hypertension). The questionnaire was encrypted and completed under medical staff's instruction.

\section{Sampling}

A sterile calcium alginate swab was used to obtain samples from patient's lower conjunctival sac for the operation (OP) and non-OP eyes separately before applying topical medication for preoperative preparation. The sampling procedure was performed by trained medical staff to avoid any act, such as contact with eyelids or eyelashes, that may cause contamination, and the swab samples were immediately placed on 5\% sheep blood and chocolate agar plates and sent to the CGMH laboratory. The culture plates were incubated at $37^{\circ} \mathrm{C}$ for 3 days. During postoperative clinical follow-up, sampling was performed using the same method at 1 month, 3 monthsand 6 months after the surgery at our clinic.

\section{Isolate identification and antimicrobial susceptibility test}

CNS was identified by performing the morphology examination, gram staining and coagulase tests of grown strains. The disk diffusion method for testing the susceptibility of isolates to antibiotics, namely clindamycin, erythromycin, oxacillin, penicillin, teicoplanin and trimethoprim/ sulfamethoxazole (TMP-SMX), was performed in CGMH laboratory in accordance with guidelines published by the Clinical and Laboratory Standard Institute. ${ }^{16}$ We used Etest (bioMérieux, Inc, France) to test the susceptibility of fluoroquinolones (including ciprofloxacin, levofloxacin, gatifloxacin and moxifloxacin), tobramycin and vancomycin. The resistance of antibiotics was defined based on the minimum inhibitory concentration, and interpretive criteria from the manufacturer were followed.

\section{Patient and public involvement}

Patients or public was not involved in the design of the study.

\section{Statistical analysis}

Patient's characteristics at baseline with and without CNS colonisation were compared using Fisher's exact test for categorical variables and the t-test for age. The statistic value of t-test was adjusted to the $\mathrm{df}$ using the Welch-Satterthwaite method since the group variances were unequal. Longitudinal changes in the proportions of total colonisation and CNS colonisation were analysed using the generalised linear mixed model (GLMM) with a threelevel data structure: patient, eyes and repeated measures. We chose to employ GLMM primarily because that it can be easily used for handling the data with multilevels, especially those with three or more levels. The GLMM assumed the correlation between repeated measures to be the same. Due to all the response variables were binary, the distribution was binomial, and the link function was logit in the GLMM setting. The GLMM used two random intercepts. The first was to account for correlations between the repeated measures for an eye, and the second was to account for correlations between the two eyes for a patient. Primary independent variables in the GLMM included group (OP eyes vs non-OP eyes), time (treated as categorical variable) and group $\times$ time interaction. Within the GLMM with interaction effect, tests of contrasting main effects included two aspects. The first was to test the group difference (ie, OP eyes vs non-OP eyes) at each time point, and the second was to determine the difference between baseline and follow-up in either group. In the aforementioned analysis of contrasting main effects, Bonferroni adjustment of multiple testing 
Table 1 The demographic characteristics the patients classified by conjunctiva coagulase-negative Staphylococcus (CNS) colonisation at baseline

\begin{tabular}{lllll}
\hline \multicolumn{5}{l}{ CNS colonisation in either eye } \\
\hline Variable & Total $(\mathbf{n}=\mathbf{1 2 8})$ & No $(\mathbf{n}=\mathbf{9 7})$ & Yes $(\mathbf{n}=\mathbf{3 1})$ & $\mathbf{P}$ \\
\hline Male & $60(46.9)$ & $45(46.4)$ & $15(48.4)$ & $>0.99$ \\
Age (year, mean \pm SD) & $68.3 \pm 10.7$ & $67.5 \pm 10.8$ & $70.5 \pm 9.9$ & 0.17 \\
Hospitalisation in 1 year & $30(23.4)$ & $24(24.7)$ & $6(19.4)$ & 0.63 \\
ESRD on dialysis & $6(4.7)$ & $6(6.2)$ & $0(0.0)$ & 0.34 \\
Operation in previous & $30(23.4)$ & $27(27.8)$ & $4(12.9)$ & 0.15 \\
6 months & & & & \\
Lived with child $<5$ years & $27(21.1)$ & $23(23.7)$ & $4(12.9)$ & 0.31 \\
Skin disease & $14(10.9)$ & $10(10.3)$ & $4(12.9)$ & 0.74 \\
Liver disease & $8(6.3)$ & $7(7.2)$ & $1(3.2)$ & 0.68 \\
Diabetes mellitus & $33(25.8)$ & $22(22.7)$ & $11(35.5)$ & 0.16 \\
Heart disease & $17(13.3)$ & $12(12.4)$ & $5(16.1)$ & 0.56 \\
Chronic kidney disease & $8(6.3)$ & $5(5.2)$ & $3(9.7)$ & 0.40 \\
Hypertension & $66(51.6)$ & $47(48.5)$ & $19(61.3)$ & 0.22
\end{tabular}

Data were expressed as number and percentage; no patients had a history of using immunosuppressants, alcohol abuse, homelessness, HIV infection or malignancy.

$P$ value was obtained by Fisher's exact test, except the one for age by t-test.

ESRD, end-stage renal disease.

was made. The alpha levels of between-group difference and within-group difference were adjusted to 0.01125 $(0.05 / 4$; four time points) and $0.0083(0.05 / 6$; six pairwise comparison among four time points), respectively. Similar analyses were conducted of the longitudinal change in the antibiotic resistance rate in CNS-isolated eyes. The GLMM was repeated for all types of antibiotic in the CNS-isolated eyes. The significant effect in each GLMM model was associated to a statistic value, the $\mathrm{z}$ value, of which a value of $>1.96$ indicated statistical significance. A $p$ value $<0.05$ was considered statistically significant. All statistical analyses were performed using a commercial software (IBM SPSS V.22).

\section{RESULTS}

\section{Demographic characteristics}

A total of 128 patients who received cataract surgery were enrolled. Before cataract surgery, $54(42.2 \%)$ and $31(24.2 \%)$ patients had any bacteria and CNS colonisation, respectively, in either eye. Patient with CNS colonisation was defined as having CNS colonisation in at least one eye. The demographic characteristics are detailed in table 1 . The mean age of the patients was $68.3 \pm 10.7$ years, and $60(46.9 \%)$ of them were male. No significant difference was observed in baseline characteristics between the patients with and without CNS colonisation. No patients had postoperative infection.

\section{Colonisation}

After cataract surgery, 77, 60 and 49 patients visited the clinic for 1-month, 3-month and 6-month follow-up, respectively. Any bacterial colonisation was identified in $28(21.9 \%), 27(35.1 \%), 18(30 \%)$ and $18(36.7 \%)$ OP eyes and $40(31.3 \%), 27(35.1 \%), 21(35 \%)$ and $22(44.9 \%)$ non-OP eyes preoperatively and 1,3 and 6 months postoperatively, respectively. CNS colonisation was identified in $14(10.9 \%), 16(20.8 \%), 8(13.3 \%)$ and $10(20.4 \%)$ OP eyes and $23(18.0 \%), 17(22.1 \%)$, $13(21.7 \%)$ and $13(26.5 \%)$ non-OP eyes preoperatively and 1, 3 and 6 months postoperatively, respectively. In the OP eyes with positive colonisation at the baseline, 29, 23 and 15 eyes were followed, and 12, 7 and 7 eyes had CNS colonisation at postoperative 1, 3 and 6 months, respectively. There were 26 eyes had more than one CNS positive sampling and three eyes had persistent CNS colonisation from preoperation to all postoperative sampling (details in the supplementary table 1.) Figure 1 illustrates longitudinal changes in all bacterial colonisation and CNS colonisation in the OP and non-OP eyes during the 6-month study period. No significant difference was observed in the proportions of bacterial and CNS colonisation between the OP and non-OP eyes from baseline to a specific follow-up (all $\mathrm{p}$ values for interaction $>0.10$ ). The proportion of colonisation of any bacteria tended to increase in the OP eyes at 1 month (estimate $[B]=0.13, \mathrm{z}=1.90, \mathrm{p}=0.06$ ) and in both the OP and non-OP eyes 6 months after cataract surgery $(B=0.16, \mathrm{z}=1.82, \mathrm{p}=0.07 ; B=0.15, \mathrm{z}=1.82, \mathrm{p}=0.07$ respectively), although this was not significant. An increased trend of CNS colonisation was only observed in the OP eyes 1 month postoperatively $(B=0.09, \mathrm{z}=1.87, \mathrm{p}=0.06)$, but it was not significant. 

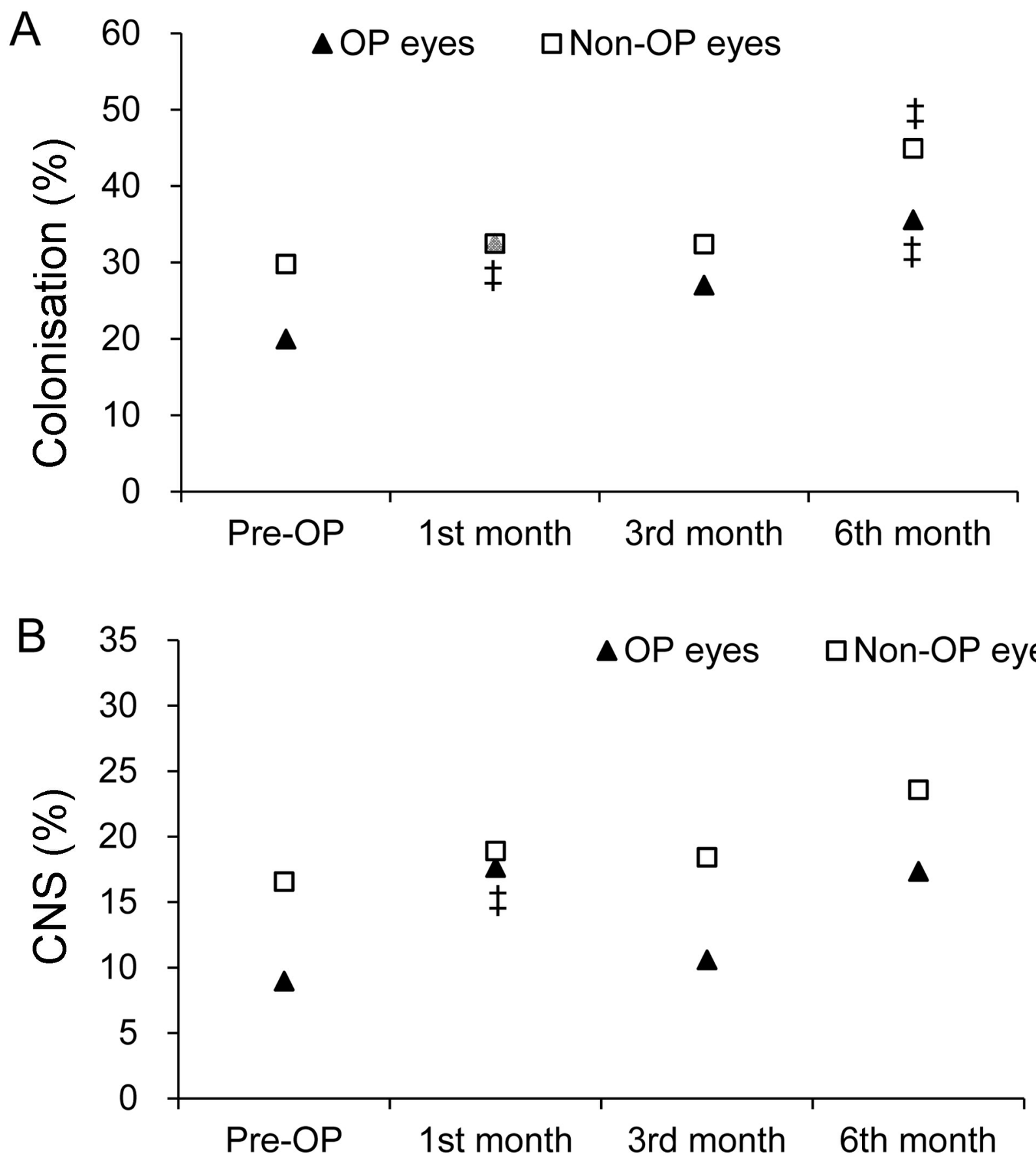

Figure 1 Estimated proportion of total colonisation (A) and coagulase-negative Staphylococcus (CNS) colonisation (B) on operation (OP) and non-OP eyes through 6 month follow-up. *Indicates the Bonferroni-adjusted $p<0.10$ (unadjusted $\mathrm{P}<0.017)$ compared with pre-OP within group. The OP eyes and non-OP eyes were simultaneously estimated in a GLMM analysis. GLMM, generalised linear mixed model.

\section{Antibiotic resistance of CNS}

Longitudinal changes in the antibiotic resistance of CNS are shown in figure 2. Before cataract surgery, fluoroquinolone resistance rates ranged from $8.7 \%$ to $20 \%$, and tobramycin resistance was approximately $47 \%$ in both the eyes (details are shown in table 2). In the OP eyes, a significant increase in antibiotic resistance was found to tobramycin at 1 month and to ciprofloxacin at 3 months $(B=0.37$, $\mathrm{z}=2.28, \quad \mathrm{p}=0.03 ; \quad B=0.43, \quad \mathrm{z}=2.14, \quad \mathrm{p}=0.04$, respectively $)$.
Tobramycin resistance was substantially higher in the OP eyes than in the non-OP eyes at 1 month $(81 \%$ vs $47 \%$; $B=0.35, \mathrm{z}=2.08, \mathrm{p}=0.04)$. In the non-OP eyes, significant increases in antibiotic resistance were found to TMP-SMX at 1 and 3 months $(B=0.24, \mathrm{z}=1.94, \mathrm{p}=0.06 ; B=0.27, \mathrm{z}=1.81$, $\mathrm{p}=0.08$, respectively $)$ and to oxacillin at 6 months $(B=0.45$, $\mathrm{z}=3.14, \mathrm{p}=0.003)$. Although most of the results did not reach statistical significance, increased resistance after surgery to most of the antibiotics was observed in both 

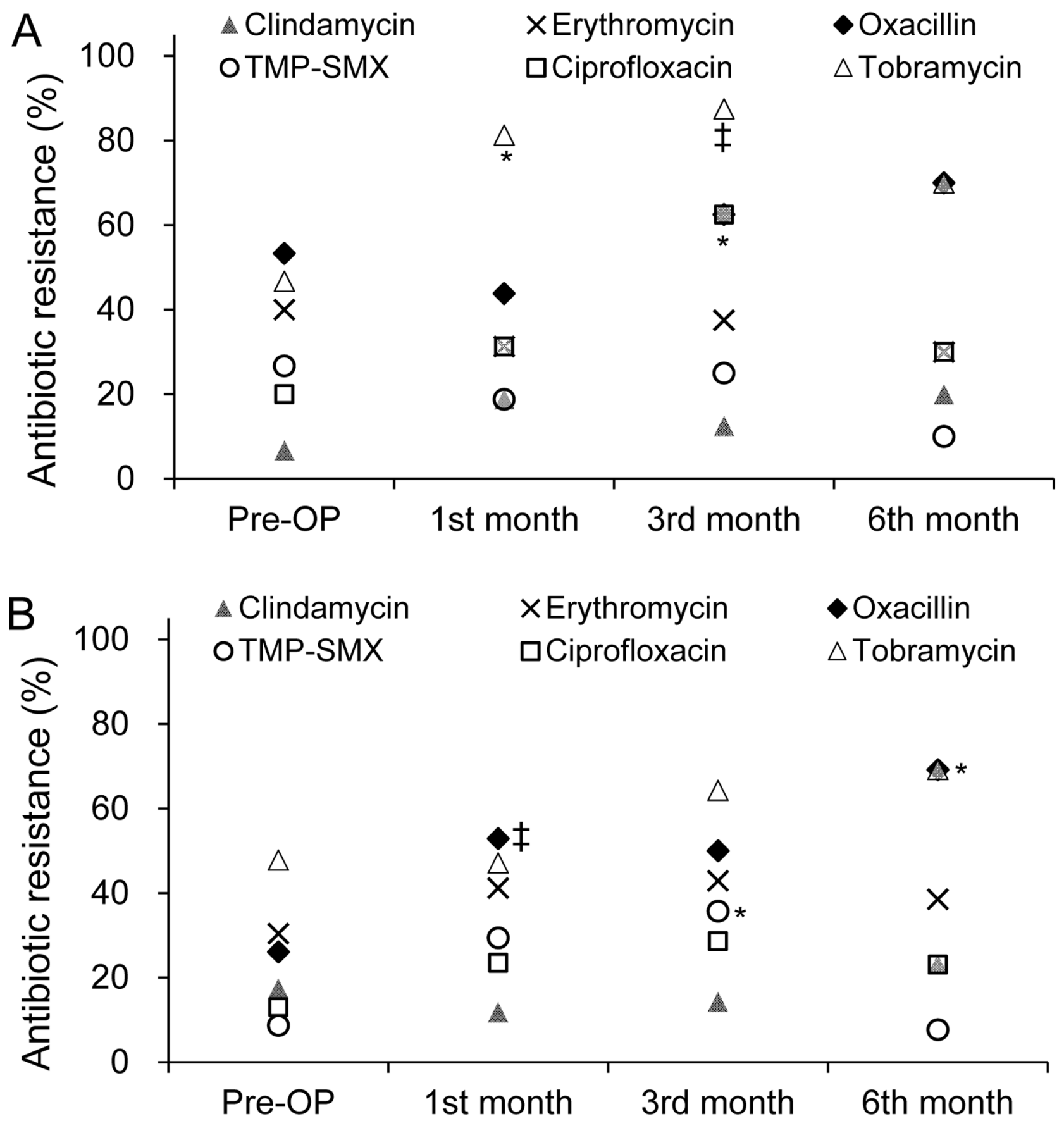

Figure 2 Changes in antibiotic resistance response of coagulase-negative Staphylococcus (CNS) isolated from operation (OP) eyes $(A)$ and non-OP eyes $(B)$. *Indicates the Bonferroni-adjusted $p<0.05$ (unadjusted $p<0.008$ ) compared with pre-OP within group. ${ }^{+1}$ ndicates the Bonferroni-adjusted $p<0.10$ (unadjusted $p<0$. 017) compared with pre-OP within group. The OP eyes and non-OP eyes were simultaneously estimated in a GLMM analysis. The six antibiotic resistance responses were analysed separately in six GLMMs. GLMM, generalised linear mixed model; TMP-SMX, trimethoprim/sulfamethoxazole.

the OP and non-OP eyes (table 2). The phenomenon was especially pronounced to tobramycin in both the eyes. For eyes with more than one CNS-positive sampling, resistance to tobramycin rose from $50.0 \%$ at preoperative sampling to $71.4 \%, 84.6 \%$ and $80.0 \%$ at 1,3 and 6 months postoperatively (data not shown). Vancomycin and teicoplanin resistance were not detected in all CNS isolates during the study period.

\section{DISCUSSION}

This observational and longitudinal study analysed conjunctival CNS colonisation and the antibiotic resistance profiles of the OP and non-OP eyes after cataract surgery with prophylactic $0.3 \%$ tobramycin treatment. We found that the colonisation rates of bacteria and CNS did not decrease throughout the 6-month follow-up, and an increased trend of resistance of CNS to several antibiotics 
Table 2 Percentage of antibiotic resistance of coagulase-negative Staphylococcus (CNS) isolated from operation (OP) eyes and non-OP eyes

\begin{tabular}{|c|c|c|c|c|c|c|c|c|}
\hline \multirow[b]{2}{*}{ Antibiotic } & \multicolumn{2}{|c|}{ Pre-OP (n=128) } & \multicolumn{2}{|c|}{ First month $(n=77)$} & \multicolumn{2}{|c|}{ Third month $(n=60)$} & \multicolumn{2}{|c|}{ Sixth month $(n=49)$} \\
\hline & $\begin{array}{l}\text { OP eyes } \\
(n=14)^{\star}\end{array}$ & $\begin{array}{l}\text { Non-OP } \\
\text { eyes } \\
(n=23)\end{array}$ & $\begin{array}{l}\text { OP eyes } \\
(n=16)\end{array}$ & $\begin{array}{l}\text { Non-OP } \\
\text { eyes } \\
(n=17)\end{array}$ & $\begin{array}{l}\text { OP eyes } \\
(n=8)\end{array}$ & $\begin{array}{l}\text { Non-OP } \\
\text { eyes } \\
(n=13)^{*}\end{array}$ & $\begin{array}{l}\text { OP eyes } \\
(n=10)\end{array}$ & $\begin{array}{l}\text { Non-OP } \\
\text { eyes } \\
(n=13)\end{array}$ \\
\hline Clindamycin & 6.7 & 17.4 & 18.8 & 11.8 & 12.5 & 14.3 & 20 & 23.1 \\
\hline Erythromycin & 40 & 30.4 & 31.3 & 41.2 & 37.5 & 42.9 & 30 & 38.5 \\
\hline Oxacillin & 53.3 & 26.1 & 43.8 & $52.9 \dagger$ & 62.5 & 50 & 70 & $69.2 \ddagger$ \\
\hline TMP-SMX & 26.7 & 8.7 & 18.8 & $29.4 \ddagger$ & 25.0 & $35.7 \neq$ & 10 & 7.7 \\
\hline Ciprofloxacin & 20 & 13.0 & 31.3 & 23.5 & $62.5 \ddagger$ & 28.6 & 30 & 23.1 \\
\hline Gatifloxacin & 20 & 13.0 & 31.3 & 23.5 & 37.5 & 28.6 & 30 & 23.1 \\
\hline Levofloxacin & 20 & 13.0 & 31.3 & 23.5 & 37.5 & 28.6 & 30 & 23.1 \\
\hline Vancomycin & 0 & 0 & 0 & 0 & 0 & 0 & 0 & 0 \\
\hline
\end{tabular}

Results were derived from generalised linear mixed model.

${ }^{*}$ One eye with 2 CNS isolates identified.

tindicates Bonferroni-adjusted $p<0.10$ (unadjusted $p<0$. 017) compared with pre-OP within group.

findicates Bonferroni-adjusted $p<0.05$ (unadjusted $p<0$. 008) compared with pre-OP within group.

§indicates Bonferroni-adjusted $p<0.05$ (unadjusted $p<0.012$ ) compared with OP eyes within a specific follow-up time.

TMP-SMX, trimethoprim/sulfamethoxazole.

was observed in both the OP and non-OP eyes when compared with the preoperative condition. This finding indicates that prolonged postoperative antibiotic treatment is at a non-lethal concentration, and it may change the antibiotic profile of CNS strains in both eyes.

Antibiotic resistance profiles of CNS isolates may clarify the environmental change after topical antibiotic administration, because CNS is the most commonly isolated organism from the conjunctival sac and a main pathogen of endophthalmitis. ${ }^{11-14}$ Nejima et at investigated the effect of postoperative $1.5 \%$ levofloxacin administration on 104 patients who had undergone cataract surgery and found increased levofloxacin resistance of Staphylococcus epidermidis, which is one of the most common CNS strains. In that investigation, the resistance rate was higher in patients who received 1 month levofloxacin treatment than in patients who received 1 week levofloxacin treatment, and the resistant rate gradually declined 3 months after completion of antibiotic administration. Miyanaga $e t a l^{8}$ reported increased resistance of S. epidermidis to levofloxacin and a number of gene mutations in the quinolone resistance-determining region after $0.5 \%$ levofloxacin administration for 1 week preoperatively and 2 weeks postoperatively. Moreover, both studies found that perioperative levofloxacin eye drops failed to reduce the S. epidermidis colonisation rate after cataract surgery. ${ }^{89}$

Unlike previous longitudinal studies of conjunctival flora after cataract surgery, ${ }^{8-10}$ we retrieved samples from the OP eyes and from the non-OP eyes. Moreover, we performed antibiotic susceptibility tests for several antibiotics that are available for ophthalmic use. We found that postoperative $0.3 \%$ tobramycin administration had no significant effect on the elimination of conjunctival flora and CNS strains. Although povidone-iodine sterilisation has been performed to the OP eyes, the bacterial colonisation rate was not decreased at postoperative 1 month. It confirmed the effect of decolonisation by povidone-iodine wash only persisted for a short period of time. ${ }^{17}$ Additionally, resistance to tobramycin in the OP eyes significantly increased 1 month postoperatively, and the development of additional drug resistances, including those to oxacillin, TMP-SMX and ciprofloxacin, of CNS isolates in either eye was observed during the follow-up. The effect of multidrug resistance, defined as resistance to three or more antimicrobial categories, ${ }^{18}$ seemed to persist for at least 3 months postoperatively; this result has also been found in perioperative levofloxacin administration for cataract surgery. ${ }^{10}$ This suggests that prophylactic $0.3 \%$ tobramycin administration after cataract surgery exerts a long-term effect of selecting resistant CNS strains.

Selection of antibiotic resistance at a non-lethal antibiotic concentration has been reported and is a crucial environmental issue. ${ }^{19-21}$ A non-lethal antibiotic concentration increases the rate of selection and selectively enriches resistant mutants with higher fitness. ${ }^{19}$ Although most of the related studies have focused on the development of resistance to a single antibiotic, gene mutation for resistant strains after using certain antibiotics may also induce 
multidrug resistance. ${ }^{8}$ A longitudinal study of patients who were randomly assigned to1 of 4 different antibiotic treatments after intravitreal injection reported that repeated exposure to ophthalmic antibiotics in treated eyes increased multidrug resistance in $67.5 \%$ of CNS isolates to at least five antibiotics. ${ }^{22}$ Multidrug resistance is commonly generated through the accumulation of resistance-related genes or increased expression of multidrug efflux pumps, ${ }^{23}$ which can be related to use of a single antibiotic. This could explain the increased resistance rate to several antibiotics other than tobramycin in our study.

Our study indicated that changes in flora in either side of conjunctival sac may affect the other because of the similar pattern in the bacterial colonisation and resistance profiles in both eyes. Micro-organisms inhabit many sites of the human body, particularly the skin, and the bacterial community shares similar molecular characteristic and strain of harbouring in a specific site. ${ }^{24}$ For example, Staphylococcus aureus nostril colonisation demonstrated identical strains from skin and soft tissue infection. ${ }^{25}$ Ocular surfaces, such as the conjunctiva, have close contact with the eyelid and the skin; therefore, both eyes are likely to share similar microbial colonisation indirectly through the connecting skin. However, our study lacked molecular analysis of microbiological genotypes and was unable to ascertain the similarity of bacterial strains from the colonisation from the two conjunctival sacs.

The baseline fluoroquinolone resistance rate of CNS in our study (15\%) was lower than that in Japan (26\%$47 \%),{ }^{8} 926$ where fluoroquinolone agents are commonly used. The National Health Insurance Administration in Taiwan reserves fluoroquinolones for the treatment of severe bacterial infections. Conversely, the tobramycin resistance rate of CNS strains was relatively higher in our study $(47 \%)$ than in other comparable studies (36\% in Italy ${ }^{27}$ and $25 \%$ in Uganda), ${ }^{28}$ because tobramycin is the most commonly used ophthalmic antibiotic in our hospital. ${ }^{13}$ Antibiotic preference and selective antibiotic pressure result in different susceptibility in different regions. It is difficult to explore the association among conjunctival colonisation, tobramycin use and endophthalmitis, because there was no postoperative infection in our study. Antibiotic administration after cataract surgery is commonly used in Taiwan. In our hospital, preoperative povidone-iodine wash and postoperative antibiotic administration have been practised for more than 20years for preventing postoperative endophthalmitis. Nevertheless, this study found that postoperative antibiotic administration failed to reduce conjunctival flora but induced antibiotic resistance. For preventing endophthalmitis after cataract surgery, although no such case occurred in this study, the current routine in our hospital should be reconsidered. In randomised endophthalmitis prophylaxis trials by the European Society of Cataract and Refractive Surgeons, intracameral cefuroxime injection resulted in a nearly fivefold decrease in the risk of endophthalmitis after cataract surgery. ${ }^{29}{ }^{30}$ Recently, intraoperative intracameral antibiotic injection is gaining popularity for preventing endophthalmitis after cataract surgery. ${ }^{5}$
This study has some limitations. First, the major limitation is that small number of cases and low bacterial yield in the study may have affected statistical significance. ${ }^{13}$ The drop-out rate in the follow-up period is relatively high. However, significant differences were still found in the study and may give a reference for future clinical practices. Second, not all patients attended the scheduled visits after the operation, and the missing follow-up may also have influenced the results. Third, we did not perform molecular analysis for isolates from the conjunctival sac; therefore, we were unable to compare genetic relatedness with the colonisation isolates. The strengths of this study are as follows. Unlike previous studies, we performed sampling for both the eyes separately to provide an internal control from the non-OP eyes. Furthermore, we examined drug resistance to tobramycin and performed susceptibility tests for several antibiotics that can be prescribed for ophthalmic use. Lastly, few studies have analysed longitudinal changes in conjunctival colonisation. We followed the patients until 6 months after cataract surgery in the study. Although missing follow-ups may have affected significance, the results provide meaningful information by showing a microbiological profile of the OP and non-OP eyes during the study period.

In conclusion, this study reported longitudinal changes in CNS colonisation and its antibiotic susceptibility after cataract surgery. Postoperative $0.3 \%$ tobramycin administration was at a non-lethal concentration and may increase the resistance of CNS strains to multiple antibiotics in both the OP and non-OP eyes. Evidence endorses prophylactic routines, such as intracameral antibiotic injection, that are preferable for preventing endophthalmitis after cataract extraction.

Contributors The author contributions were as followed: conception and design: $\mathrm{Chin}-\mathrm{HH}$ and $\mathrm{Y}-\mathrm{CH}$; sample and data collection: Chiu-HH; analysis and interpretation: EY-CK, Chin-HH, Y-CH; writing: EY-CK; overall responsibility: Chin-HH.

Funding National Science Council, Taiwan (NSC103-2314-B-182A-043-MY2, NMRPG3D6072) and Chang Gung Memorial Hospital, Taiwan (CMRPG3F0111).

Competing interests None declared.

Patient consent for publication Obtained.

Ethics approval This study was approved by the Institutional Review Board of Chang Gung Memorial Hospital (IRB-100-1138C).

Provenance and peer review Not commissioned; externally peer reviewed.

Data sharing statement The data used for the current study cannot be made publicly available.

Open access This is an open access article distributed in accordance with the Creative Commons Attribution Non Commercial (CC BY-NC 4.0) license, which permits others to distribute, remix, adapt, build upon this work non-commercially, and license their derivative works on different terms, provided the original work is properly cited, appropriate credit is given, any changes made indicated, and the use is non-commercial. See: http://creativecommons.org/licenses/by-nc/4.0/.

\section{REFERENCES}

1. HUGHES WF, Owens WC. Postoperative complications of cataract extraction. Arch Ophthal 1947;38:577-95.

2. Taban M, Behrens A, Newcomb RL, et al. Acute endophthalmitis following cataract surgery: a systematic review of the literature. Arch Ophthalmol 2005;123:613-20. 
3. Lundström M, Friling E, Montan P. Risk factors for endophthalmitis after cataract surgery: Predictors for causative organisms and visual outcomes. J Cataract Refract Surg 2015;41:2410-6.

4. Huang J, Wang X, Chen X, et al. Perioperative Antibiotics to Prevent Acute Endophthalmitis after Ophthalmic Surgery: A Systematic Review and Meta-Analysis. PLoS One 2016;11:e0166141.

5. Kessel L, Flesner P, Andresen J, et al. Antibiotic prevention of postcataract endophthalmitis: a systematic review and metaanalysis. Acta Ophthalmol 2015;93:303-17.

6. Chang DF, Braga-Mele R, Henderson BA, et al. Antibiotic prophylaxis of postoperative endophthalmitis after cataract surgery: Results of the 2014 ASCRS member survey. J Cataract Refract Surg 2015;41:1300-5.

7. Rudnisky CJ, Wan D, Weis E. Antibiotic choice for the prophylaxis of post-cataract extraction endophthalmitis. Ophthalmology 2014;121:835-41.

8. Miyanaga M, Nejima R, Miyai T, et al. Changes in drug susceptibility and the quinolone-resistance determining region of Staphylococcus epidermidis after administration of fluoroquinolones. $J$ Cataract Refract Surg 2009;35:1970-8.

9. Nejima R, Shimizu K, Ono T, et al. Effect of the administration period of perioperative topical levofloxacin on normal conjunctival bacterial flora. J Cataract Refract Surg 2017;43:42-8.

10. Ono T, Nejima R, Iwasaki T, et al. Long-term effects of cataract surgery with topical levofloxacin on ocular bacterial flora. $J$ Cataract Refract Surg 2017:43:1129-34.

11. Wu PC, Kuo HK, Li M, et al. Nosocomial postoperative endophthalmitis: a 14-year review. Graefes Arch Clin Exp Ophthalmol 2006:244:920-9.

12. Walker $\mathrm{CB}$, Claoué $\mathrm{CM}$. Incidence of conjunctival colonization by bacteria capable of causing postoperative endophthalmitis. $J R$ Soc Med 1986;79:520-1.

13. Lin $\mathrm{YH}$, Kang $\mathrm{YC}$, $\mathrm{Hou} \mathrm{CH}$, et al. Antibiotic susceptibility profiles of ocular and nasal flora in patients undergoing cataract surgery in Taiwan: an observational and cross-sectional study. BMJ Open 2017;7:e017352.

14. Suto $\mathrm{C}$, Morinaga M, Yagi T, et al. Conjunctival sac bacterial flora isolated prior to cataract surgery. Infect Drug Resist 2012;5:37-41.

15. Speaker MG, Milch FA, Shah MK, et al. Role of external bacterial flora in the pathogenesis of acute postoperative endophthalmitis. Ophthalmology 1991;98:639-50. discussion 650

16. Clinical and Laboratory Standards Institute. M100-S25 Performance Standards for Antimicrobial Susceptibility Testing;Twenty-Fifth Informational Supplement. 2015.
17. Rossetto JD, Suwannaraj S, Cavuoto KM, et al. Evaluation of Postoperative Povidone-lodine in Adjustable Suture Strabismus Surgery to Reduce Suture Colonization: A Randomized Clinical Trial. JAMA Ophthalmol 2016;134:1151-5.

18. Magiorakos AP, Srinivasan A, Carey RB, et al. Multidrug-resistant, extensively drug-resistant and pandrug-resistant bacteria: an international expert proposal for interim standard definitions for acquired resistance. Clin Microbiol Infect 2012;18:268-81.

19. Andersson DI, Hughes D. Evolution of antibiotic resistance at nonlethal drug concentrations. Drug Resist Updat 2012;15:162-72.

20. Hughes D, Andersson DI. Selection of resistance at lethal and nonlethal antibiotic concentrations. Curr Opin Microbiol 2012;15:555-60.

21. Khan S, Beattie TK, Knapp CW. The use of minimum selectable concentrations (MSCs) for determining the selection of antimicrobial resistant bacteria. Ecotoxicology 2017;26:283-92.

22. Kim SJ, Toma HS. Ophthalmic antibiotics and antimicrobial resistance a randomized, controlled study of patients undergoing intravitreal injections. Ophthalmology 2011;118:1358-63.

23. Nikaido H. Multidrug resistance in bacteria. Annu Rev Biochem 2009;78:119-46.

24. Grice EA, Kong HH, Conlan S, et al. Topographical and temporal diversity of the human skin microbiome. Science 2009;324:1190-2.

25. Wertheim HF, Melles DC, Vos MC, et al. The role of nasal carriage in Staphylococcus aureus infections. Lancet Infect Dis 2005;5:751-62.

26. Hori Y, Maeda N, Sakamoto M, et al. Fluoroquinolone-resistant bacteria and methicillin-resistant Staphylococci from normal preoperative conjunctiva. J Cataract Refract Surg 2008:34:711-2.

27. Papa V, Blanco AR, Santocono M. Ocular flora and their antibiotic susceptibility in patients having cataract surgery in Italy. J Cataract Refract Surg 2016;42:1312-7.

28. Mshangila $\mathrm{B}$, Paddy $\mathrm{M}$, Kajumbula $\mathrm{H}$, et al. External ocular surface bacterial isolates and their antimicrobial susceptibility patterns among pre-operative cataract patients at Mulago National Hospital in Kampala, Uganda. BMC Ophthalmol 2013;13:71

29. Endophthalmitis Study Group. European Society of Cataract \& Refractive Surgeons. Prophylaxis of postoperative endophthalmitis following cataract surgery: results of the ESCRS multicenter study and identification of risk factors. J Cataract Refract Surg 2007:33:978-88.

30. Barry P, Seal DV, Gettinby G, et al. ESCRS study of prophylaxis of postoperative endophthalmitis after cataract surgery: Preliminary report of principal results from a European multicenter study. $J$ Cataract Refract Surg 2006;32:407-10. 